\title{
Mathematical simulation of heat transfer processes at the maximum possible electrical loads in typical light-emitting diodes
}

\author{
Oleg M. Rudenko and Pavel A. Strizhak \\ National Research Tomsk Polytechnic University 30, Lenin Avenue, Tomsk 634050, Russia
}

\begin{abstract}
The physical and mathematical models for prognostic research of possible thermal operating modes of typical light-emitting diodes (LED) at critical electrical loads have been developed. The highest currents have been set, at which LED's compound melting begins. LED's characteristic times at these electrical loads have been identified. The influences of compound's material properties (density, thermal conductivity, heat capacity) on LED's operating characteristics have been determinate at critical currents loads.
\end{abstract}

\section{Introduction}

Today LED technology becomes widespread (especially it should be noted during the last decade) in many areas of human activity. The rapid rearmament of manufacturing led to a strong dependence of many engineering systems on reliability of LEDs functioning. The statistics shows that LEDs are characterized by quite low parameters of reliability. As a rule, their functional loss is a consequence of overloads, short circuits and deformations [1]. The processes regularities analysis allows making a conclusion describing, first of all, a regulation conditions disruption of LED heat exchange with the environment and, as a result of it, the LED fails [2, 3].

There are several methods to analyze the thermal calculations of LED operation (for example, the calculation based on thermal models [4], research of a LED's characteristics in thermal cameras [5], etc.). The balanced models and software applications based on them (the LED's thermal resistance calculation models [2], taking into account the infrared radiation effects [6], p-n junction temperature change [4], as well as CFD-simulation [7]) are the mostly widely used. However, many experiments $[3,5,6,8,9]$ with different LEDs showed that the balanced models application domain is very limited. Particularly, the balanced models provide the approximate, quite significantly different from the real, processes at an intensive heat release or an energy absorption due to the chemical reactions and phase transformations (melting, crystallization).

Balanced models provide the LEDs operation integral characteristics, significantly (over 20\%) different from experimental ones. It is expediently to create the complex physical and mathematical models of conjugate heat transfer with the main factors and processes affecting the LED's operation.

\footnotetext{
${ }^{\text {a }}$ Corresponding author: pavelspa@tpu.ru
}

This is an Open Access article distributed under the terms of the Creative Commons Attribution License 4.0, which permits unrestricted use, distribution, and reproduction in any medium, provided the original work is properly cited. 


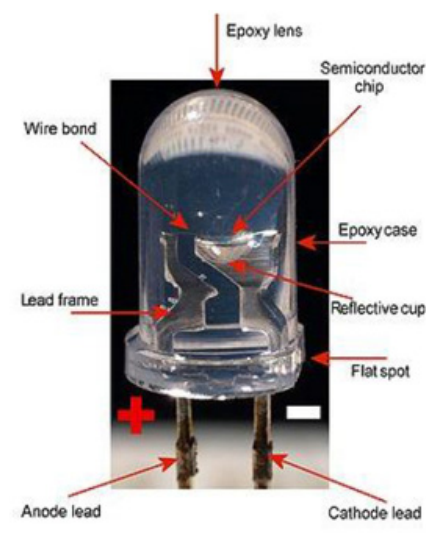

a)

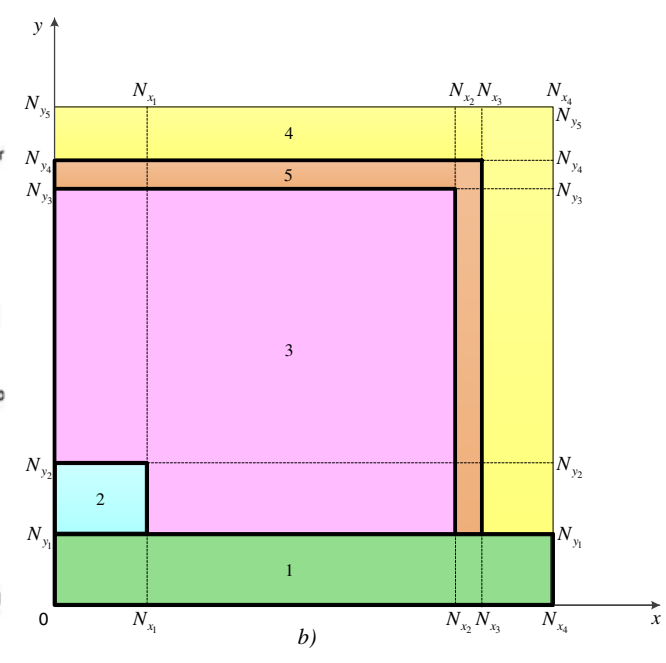

Figure 1. Scheme of a typical indicated light-emitting diode (a) and solution domain scheme for the simulation of heat transfer problem (b): 1 - substrate, 2 - crystal, 3 - compound in lens, 4 - ambient air, 5 - lens.

The work aim is the mathematical simulation of interdependent heat transfer processes under the influence of maximum possible typical LEDs' electrical loads.

\section{Physical heat transfer model}

At the physical formulation of the heat transfer problem the following processes have been considered. Energy in the crystal is released in the radiation form when the current flows through the p-n junction. The crystal is heated. Thermal radiation flux does not pass to the substrate and the rays are not reflected due to the reflector. Focused by the lens, the radiation fluxes from crystal are generated. The substrate and optically transparent polymeric compound-filled enclosure (lens) are heated by thermal conduction and radiation. The heating level of crystal, substrate and the focusing lens increases with time. In extreme cases it can lead to the crystal melting and subsequent light-emitting diodes functional loss. The problem has been formulated for a typical indicator LED (Fig. 1a).

One of the problems of theoretical investigation is the establishment of limit values for the currents and operating time of LED without its basic elements overheating. Natural and laboratory experiments demand heavy expenses of time and material resources. Therefore, it is appropriate to conduct the numerical simulation of the heat transfer processes.

The axisymmetric formulation of the heat transfer problem was being concerned (Fig. 1b). Two mechanisms of a heat transfer in LED were considered - conductive and radiant. Refraction, absorption, scattering and focusing of rays by the lens and the thermal and physical characteristics dependence on the temperature were not taking into account. The conductive heat transfer mechanism was simulated for the ambient air area of LED.

Also it was assumed during the simulation that the space inside the lens is filled with a homogeneous extender - compound with constant thermal and physical characteristics. This assumption is typical for the heat transfer processes in the optical elements and does not impose the significant restrictions on solidarity in the problem statement.

Figure 1a shows the account of different LED's configuration is the most difficulty during heat and mass transfer processes simulation. Algorithm implementation of numerical solution envisages rather laborious conjugation of spherical (polar) and cylindrical coordinate grids [10]. Sometimes sufficiently 
composite models allow obtaining the results that are insignificant (to 20\%) differ from more simple models with a Cartesian coordinate system. In addition, the form of a reflector, width of crystal "forbidden" zone and its variation with temperature increase imply the most significant role. Therefore, during numerical simulation some few simplified scheme with a Cartesian coordinate system was used to.

\section{Mathematical model and solution methods}

System of nonstationary differential equations describing the set of interrelated heat transfer processes occurring in "LED's substrate - crystal - compound - lens - ambient air" system, in accordance with the stated physical problem statement is as follows.

Thermal conductivity equation for the substrate:

$$
\rho_{1} C_{1} \frac{\partial T_{1}}{\partial t}=\lambda_{1}\left(\frac{\partial^{2} T_{1}}{\partial x^{2}}+\frac{1}{x} \frac{\partial T_{1}}{\partial x}+\frac{\partial^{2} T_{1}}{\partial y^{2}}\right), 0<x<N_{x_{4}}, 0<y<N_{y_{1}} .
$$

Thermal conductivity equation for the crystal:

$$
\rho_{2} C_{2} \frac{\partial T_{2}}{\partial t}=\lambda_{2}\left(\frac{\partial^{2} T_{2}}{\partial x^{2}}+\frac{1}{x} \frac{\partial T_{2}}{\partial x}+\frac{\partial^{2} T_{2}}{\partial y^{2}}\right)+\frac{Q}{V_{c r} t}, \quad 0<x<N_{x_{1}}, N_{y_{1}}<y<N_{y_{2}} .
$$

Thermal conductivity equation for the compound:

$$
\rho_{3} C_{3} \frac{\partial T_{3}}{\partial t}=\lambda_{3}\left(\frac{\partial^{2} T_{3}}{\partial x^{2}}+\frac{1}{x} \frac{\partial T_{3}}{\partial x}+\frac{\partial^{2} T_{3}}{\partial y^{2}}\right), 0<x<N_{x_{1}}, N_{y_{2}}<y<N_{y_{3}} ; 0<x<N_{x_{4}}, N_{y_{4}}<y<N_{y_{5}} .
$$

Thermal conductivity equation for the ambient air:

$$
\begin{aligned}
\rho_{4} C_{4} \frac{\partial T_{3}}{\partial t} & =\lambda_{4}\left(\frac{\partial^{2} T_{4}}{\partial x^{2}}+\frac{1}{x} \frac{\partial T_{4}}{\partial x}+\frac{\partial^{2} T_{4}}{\partial y^{2}}\right), \\
N_{x_{3}}<x & <N_{x_{4}}, N_{y_{1}}<y<N_{y_{5}} ; N_{x_{1}}<x<N_{x_{2}}, N_{y_{1}}<y<N_{y_{3}} .
\end{aligned}
$$

Thermal conductivity equation for the LED's lens:

$$
\begin{aligned}
\rho_{4} C_{4} \frac{\partial T_{3}}{\partial t} & =\lambda_{4}\left(\frac{\partial^{2} T_{4}}{\partial x^{2}}+\frac{1}{x} \frac{\partial T_{4}}{\partial x}+\frac{\partial^{2} T_{4}}{\partial y^{2}}\right), \\
0<x & <N_{x_{3}}, N_{y_{3}}<y<N_{y_{4}} ; N_{x_{2}}<x<N_{x_{3}}, N_{y_{1}}<y<N_{y_{4}} .
\end{aligned}
$$

Here:

$x, y$-coordinates of the cylindrical coordinate system, $\mathrm{m}$;

$t$ - time, s;

$\rho-$ density, $\mathrm{kg} / \mathrm{m}^{3}$

$T$ - temperature, $\mathrm{K}$;

$C$ - specific heat capasity, $\mathrm{J} /(\mathrm{kg} \cdot \mathrm{K})$;

$\lambda$ - thermal conductivity coefficient, $\mathrm{W} /(\mathrm{m} \cdot \mathrm{K})$;

$V_{c r}$ - volume of the crystal, $\mathrm{m}^{3}$;

$Q$ - amount of heat generated in the crystal during the electric current flow, J.

According to Joule's law the amount of heat, generated in the crystal during the electric current flow, can be determined by the following equation:

$$
Q=I^{2} R t
$$

where: $I$-current intensity, $A ; R$ - electrical resistance, Ohm; $t$ - time, s. 
Table 1. Thermal and physical characteristics of the LED's main elements.

\begin{tabular}{|l|c|c|c|}
\hline Medium (material) & $\begin{array}{c}\text { Thermal capacity, } \\
\mathrm{J} /(\mathrm{kg} \cdot \mathrm{K})\end{array}$ & $\begin{array}{c}\text { Density, } \\
\mathrm{kg} / \mathrm{m}^{3}\end{array}$ & $\begin{array}{c}\text { Thermal conductivity, } \\
\mathrm{W} /(\mathrm{m} \cdot \mathrm{K})\end{array}$ \\
\hline Crystal (Gallium nitride) & 452 & 6150 & 130 \\
\hline Compound (Organic silicone) & 1300 & 1160 & 0,2 \\
\hline Substrate (Silicon carbide) & 670 & 3220 & 370 \\
\hline Lens (Plexiglas) & 1270 & 1190 & 0.19 \\
\hline Air & 1.006 & 1190 & 0.026 \\
\hline
\end{tabular}

\section{Basic data}

The left and right bounds temperature was taken equal to $313 \mathrm{~K}$, compound melting temperature was taken equal to $450 \mathrm{~K}$. The most typical (Table 1) materials were used for the main elements of LED:

The equations system (1)-(5) with the appropriate initial and boundary conditions has been solved by the finite difference method [11]. The locally-one method has been used [11] to solve the difference analogues of the differential equations The nonlinear difference analogues of the differential equations has been solved by the iteration method [11]. The sweep method with using a implicit four-point difference scheme has been applied [11] to solve the one-dimensional difference equations.

The irregular time step of $1 \cdot 10^{-5} \mathrm{~s}$ has been used to improve the accuracy and reduce the amount of computations. The calculations have been provided by using transformed spatial grid. This algorithm is similar to the previously-described [10]. The area with sizes $l=2,5 \cdot 10^{-3} \mathrm{~m}$ and $h=4 \cdot 10^{-3} \mathrm{~m}$ has been chosen [8] to analyze the process of heating the LED components. Coordinate grids equal to 200 nodes for each coordinate have been applied.

The reliability of the received results has been determined by checking the conservatism of difference schemes, its algorithm is presented in [10].

\section{Results and discussions}

LED crystal and compound, providing the different impacts protection and LED's throughput, are considered as the main investigation object. Local overheating, the degradation (interstices) formation and, as a consequence, the temperature increase in the junction areas occur in the p-n junction when currents exceeding the rated values. It significantly influences on the LED's lifetime and its luminous flux [10]. Therefore it is necessary to identify the time dependences of exceeding the maximum acceptable temperature of the compound and the crystal operation.

It is well-known that the temperature rise of the compound operating range significantly influences on LED's colorimetric and other characteristics [3]. Taking into account the fact that the crystal melting temperature on silicon carbide substrate is above $2500 \mathrm{~K}$ [8], it is necessary to pay attention to shortcircuit currents influence on the compound area, located in the immediate vicinity of the crystal. For this the issue LED current has been varied in the range from 0.1 to $1.0 \mathrm{~A}$. Figure 2 shows the typical simulation results at time $t=0.0114 \mathrm{~s}$, the ambient temperature $T=13 \mathrm{~K}$ and the current $I=1 \mathrm{~A}$.

The possible achieve area of compound overheating limiting temperatures has been shown in Fig. 2 . The maximum heating can occur in the area near LED crystal, and, as a consequence, the compound heating near the crystal will be faster.

Figure 3 represents the dependence of the compound area overheating time near the crystal. As seen from Fig. 3 the LED functional loss temperature is reached when the current step is minimal in time (more than in 2 times from the maximum allowable value) in the compound area near the crystal.

The compound overheating temperature near the crystal reaches the limit values during a time interval less than 0.01 seconds at currents above $500 \mathrm{~mA}$ as it is shown on dependence (Fig. 3). It should be appreciated, that overheating is also reached during sufficiently short time intervals (from 0.002 to $0.07 \mathrm{~s}$ ) at currents less than $500 \mathrm{~mA}$. However, conditions of compound overheating are not reached at 

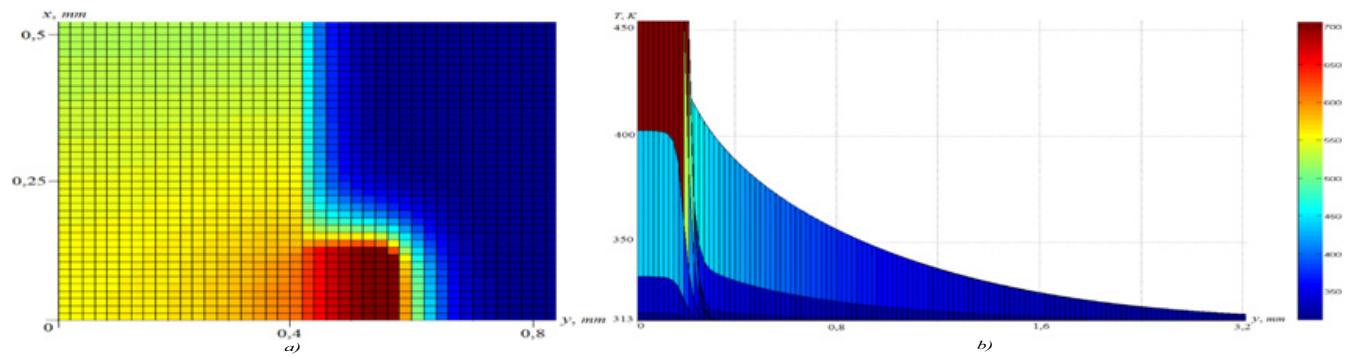

Figure 2. Isotherms (a) and temperature profile (b) at time $t=0.0048 \mathrm{~s}$, ambient temperature $T=313 \mathrm{~K}$ and current $I=1 \mathrm{~A}$.

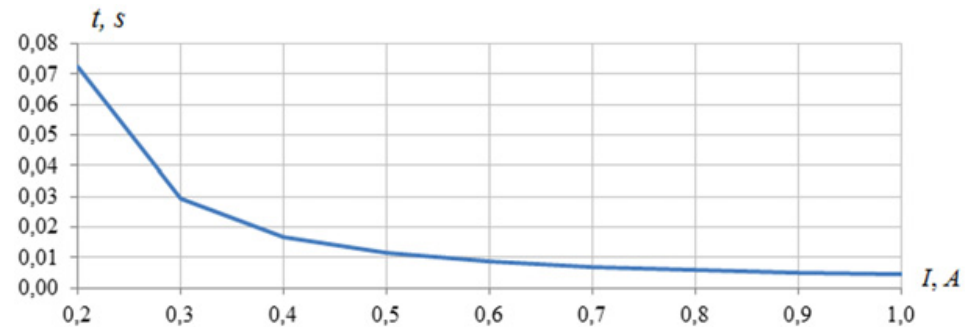

Figure 3. Dependence of the compound overheating time on the current (current change is varying from 0.1 to $1.0 \mathrm{~A})$.

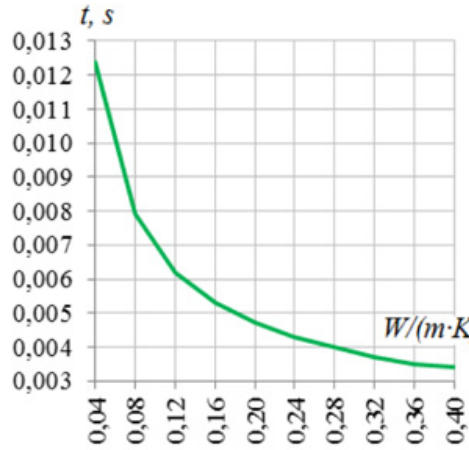

a)

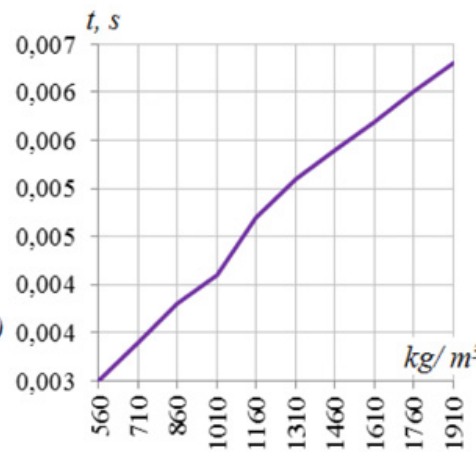

b)

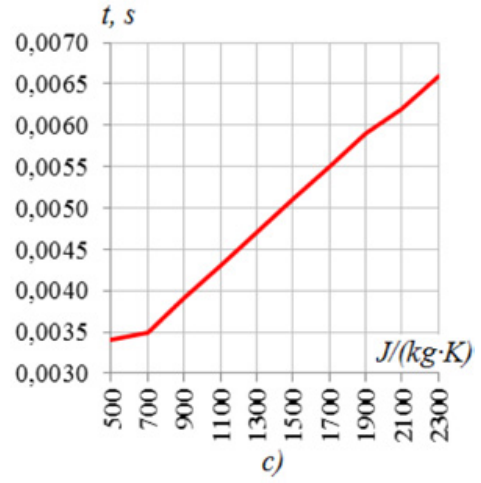

Figure 4. Time dependences of compound area overheating near to crystal at different thermal and physical characteristics (density, heat conductivity, thermal capacity) and the current force $1 \mathrm{~A}$.

currents less than $100 \mathrm{~mA}$. But there are temperatures values close to the maximum permissible, which may affect the LED light-emission characteristics.

The thermal and physical characteristics dependences on time have been obtained at current equals to 1 A to analyze the thermal and physical characteristics influences (heat conductivity, thermal capacity, density) on LED elements heating.

It may be noted, that the variation of compound density, thermal capacity and thermal conductivity influence on its time overheating. Figures $4 \mathrm{a}$ and $4 \mathrm{c}$ show that the more the density and thermal capacity of compound the more slowly the overheating is. The heating happens more quickly with the rise of 
thermal conductivity (Fig. 4b). It is possible to make a conclusion under the received dependences that the system operating period even with the proper short circuit current modes can be improved due to variation of one of the thermal and physical parameters.

It is necessary to understand that the reliability analysis model with the constrained parameters of processes in LED should be developed before engineering the production LED model. In most cases reliability analysis comes down to thermal mode studying of LED operation [1, 4-7, 9], and the LED efficiency is defined by crystal p-n junction temperature [9] being depended on the current in semiconductor [15]. The analysis of thermal and physical characteristic variation influence allows identifying model's temperature dependences and avoiding the wasted efforts during the LED production and will reduce the organization expense.

Such approach of LED heat transfer processes study allows using it sufficiently versatile to investigate the LEDs of different construction and also various factor's and load's influence. The mathematical formulation of necessary criterion which can be operationally vary according to conditions necessary for investigation is enough.

\section{Conclusion}

The developed physical and mathematical models can be used to determine the LED operation maximal currents with different environment conditions. Also it can be used for determining the compound's necessary and sufficient characteristics (thermal capacity, thermal conductivity, density) at various maximal currents and constant exterior temperature values.

This work was financially supported by the government grant "Science" (project No. 2.1321.2014).

\section{References}

[1] H. Chen, A. Keppens, P. Hanselaer, Y. Lu, Y. Gao, R. Zhuang, Z. Chen, Semiconductors, 46, 1310 (2012)

[2] M. Arik, C. A. Becker, S. E. Weaver, J. Petroski, SPIE, 5187, 64 (2004)

[3] M. Meneghini, L.-R. Trevisanello, U. Zehnder, T. Zahner, U. Strauss, G. Meneghesso, E. Zanoni, IEEE Trans. Electron. Dev., 53, 2981 (2006)

[4] C. Ma, H. Zhang, K. Cui, M. Yao, Z. Xu, ICSAI, 1596 (2012)

[5] E. Hong., N. Narendran, SPIE, 5187, 93 (2004)

[6] A. L. Zakgeim, G. L. Kuryshev, M. N. Mizerov, V. G. Polovinkin, I. V. Rozhansky, A. E. Chernyakov, Semiconductors, 44, 373 (2010)

[7] J. Chen, Z. Chen, X. Li, Y. Shen, J. Xiong, Y. Wang, ICCSEE, 2853 (2013)

[8] A. Powell, J. Jenny, S. Muller, H. McD. Hobgood, V. Tsvetkov, R. Leonard, C. Carter Jr., Int. J. High Speed Electron. Syst., 16, 751 (2006)

[9] S. Chhajed. SPIE, 5739, 16, (2005)

[10] G. V. Kuznetsov, P. A. Strizhak. Russian J. of Phys. Chem. 4, 249 (2010)

[11] A. A. Samarskii, The Theory of Difference Schemes (Marcel Dekker, Inc., USA, 2001)

[12] Y. S. Touloukian, C. Y. Ho, Thermophysical Properties of Matter (Plenum Press, New York, 1972)

[13] J. W. Chung, K. Ryu, B. Lu, T. Palacios, ESSDERC, 52 (2010)

[14] I. Akasaki, Proceedings of the IEEE, 101, 2200 (2013)

[15] M. Cooke, Semiconductor today, 7, 86 (2012) 